\title{
Can telerehabilitation deal with cognitive disturbances in epilepsy?
}

\author{
Anna Rita Giovagnoli ${ }^{1}$ (D)
}

Received: 31 May 2020 / Accepted: 19 September 2020 / Published online: 24 September 2020

(C) Fondazione Società Italiana di Neurologia 2020

Dear Editor,

More than 50\% of adult patients with epilepsy suffer cognitive disturbances. The phenotyping of temporal lobe epilepsy (TLE) puts in evidence selective memory impairments or memory impairment associated with lexical, attentive or executive deficits [1], while an involvement of higher-order control functions seems to characterize frontal lobe epilepsy [2]. These disturbances may be independent of seizures and antiepileptic drugs, may precede seizure onset and may persist after seizure control and end of treatment. Moreover, 27-64\% of patients with TLE show cognitive decline in a few years [3].

In contrast, there are few randomized, controlled or observational studies of cognitive rehabilitation in epilepsy [4]. This may depend on the prominent or exclusive importance given to seizures management and the few hospital structures dedicated to the treatment of cognitive disabilities in epilepsy. Indeed, most of the clinical and research resources are dedicated to seizure control. Additional reasons for poor application of cognitive rehabilitation may be the variability of the deficits over the course of the disease, poor patients' motivation to be engaged in this treatment, low self-efficacy and limited access to the hospital consequent to fear of unexpected seizures or incapacity to move due to lack of driving license, public transport or help from other people. Such a situation is in stark contrast to dementia, brain injury or tumour conditions where cognitive rehabilitation has been applied for a long time [5].

In this framework, the question arises whether telerehabilitation can help to face the cognitive disturbances in epilepsy. Telerehabilitation has been developed a few decades ago aiming to overcome practical problems, such as a patient's difficulty to reach the hospital, the lack of specialized centres in a patient's place of residence, excessive costs of hospital management and incongruent timing of patients' and health professionals' activities. The need for intensive or

Anna Rita Giovagnoli

annarita.giovagnoli@istituto-besta.it

1 Department of Diagnostics and Technology, Fondazione IRCCS Istituto Neurologico Carlo Besta, Milano, Italy prolonged treatments not feasible in hospital, the planning of home exercises and the need to address cognitive impairment in real life have also posed indication for telerehabilitation, providing prolonged benefits to patients with neurodegenerative diseases [6]. Recently, the use of telemedicine has become more and more important to public health due to coronavirus pandemic stimulating different telecommunication and digital technologies for cognitive, behavioural and psychological therapies.

As for epilepsy, telemedicine may contribute to surpass some limits of hospital rehabilitation. Cognitive stimulation and training in a comfortable home space may avoid the stress of moving or being accompanied to the hospital and the fear of having a seizure in the external environment, limit dependence on others, reduce the interference of external stimuli and optimize time and energy. Staying at home may put in evidence real-time daily problems caused by cognitive disturbances, therefore facilitating the planning of impairment- or improvement-tailored exercises, as well as the monitoring of cognitive failures and gains. Memory, lexical, executive and theory of mind impairments may especially benefit of such a therapeutic approach privileging verbal communication. Worth to note, methodological quality is of foremost importance in the study of non-pharmacological treatment where studies sometimes fall short of this requirement. Like other techniques, telerehabilitation needs defined programs starting from comprehensive assessment of cognitive and psychobehavioural patterns, addressing a patient's needs and perspectives $[4,5]$. Moreover, either taking inspiration from pre-existing techniques or developing new procedures, telerehabilitation needs proven reliable methods.

Given the direct interaction with a health professional and personal management of new tools, telerehabilitation may enhance a patient's sense of autonomy and his/her internal locus of control, reinforcing self-esteem and the therapeutic relationship and stimulating a patient to behave as an active partner, which is highly relevant to most epilepsy conditions. This may also enhance the feeling of being cared for and represent an opportunity to face emotional aspects [7]. Despite spatial distancing, telerehabilitation brings the professional in a familial 
context, which can relax any interactions. On the other side, telerehabilitation may ask for excessive practical demands to the patients, such as the use of unknown digital apps and personal computer, accentuating his/her sense of inadequacy. Side effects caused by repeated visual and auditory stimuli, including seizures, cannot be excluded.

To conclude, the current emphasis on telemedicine suggests reflections on the treatment of cognitive disturbances in patients with epilepsy, in particular on the scarcity of controlled studies on hospital rehabilitation and the opportunities offered by telerehabilitation. Different epilepsy-related issues may be surpassed by telerehabilitation. Recent forced experience with telemedicine may pave the way for cognitive telerehabilitation in epilepsy and other neurological conditions, highlighting the importance of investigating patients' compliance to new technologies, using valid methods and determining the effects of defined telecommunication and digital tools on selective cognitive domains.

Availability of data and material Not applicable.

\section{Compliance with ethical standards}

Conflicts of interest None.

Ethics approval Not applicable.

Consent to participate Not applicable.

Consent for publication Not applicable.
Code availability Not applicable.

\section{References}

1. Elverman KH, Resch ZJ, Quasney EE, Sabsevitz DS, Binder JR, Swanson SJ (2019) Temporal lobe epilepsy is associated with distinct cognitive phenotypes. Epilepsy Behav 96:61-68. https://doi. org/10.1016/j.yebeh.2019.04.015

2. Giovagnoli AR, Tallarita GM, Parente A, Pastori C, de Curtis M (2020) The understanding of mental states and the cognitive phenotype of frontal lobe epilepsy. Epilepsia 61:747-757. https://doi.org/ 10.1111/epi.16457

3. Hermann BP, Seidenberg M, Dow C, Jones J, Rutecki P, Bhattacharya A, Bell B (2006) Cognitive prognosis in chronic temporal lobe epilepsy. Ann Neurol 60:80-87. https://doi.org/10.1002/ ana. 20872

4. Farina E, Raglio A, Giovagnoli AR (2015) Cognitive rehabilitation in epilepsy: an evidence-based review. Epilepsy Res 109:210-218. https://doi.org/10.1016/j.eplepsyres.2014.10.017

5. Wilson B (2009) Memory rehabilitation: integrating theory and practice. Guilford Press, New York ISBN: 9781606232873

6. Pitt R, Theodoros D, Hill AJ, Russell T (2019) The impact of the telerehabilitation group aphasia intervention and networking programme on communication, participation, and quality of life in people with aphasia. Int J Speech Lang Pathol 21:513-523. https://doi. org/10.1080/17549507.2018.1488990

7. Myin-Germeys I (2020) Digital technology in psychiatry: towards the implementation of a true person-centered care in psychiatry? Eur Arch Psychiatry Clin Neurosci 270:401-402. https://doi.org/10. 1007/s00406-020-01130-1

Publisher's note Springer Nature remains neutral with regard to jurisdictional claims in published maps and institutional affiliations. 